\title{
Study of induced gravity and correlation theory analysis of nuclear Synthesis chemical elements
}

\author{
Wen Bingxiong
}

(China Guangxi Mona Lisa New Materials Co., Ltd., Guangxi, 543302)

Abstract: Modern aerodynamics is the dynamics of gas propulsion. It requires precise data, material resources, manpower, and time. It is urgent to find a more suitable power source. Based on the gravitational effects of dark energy and dark matter, the cyclic process of cosmic physics black holes to odd black holes and dark energy black holes, special fusion and fission phenomena are common in the universe, and theoretical studies such as Einstein's general theory of relativity are no exception. Through the study of the source of cosmic elements, the formation environment of stellar elements, the theory and method of modern physical artificial elements, as well as atomic bombs, hydrogen bombs, and nuclear reactors to precipitate elemental products, we have designed a device which can synthesize physically stable elements and discover new chemical elements. A device capable of synthesizing physically stable elements and discovering new chemical elements is designed.

Keywords: universal gravitational waves; chemical elements; synchrotron radiation; hadrons; hydrogen; magnets

Chinese picture classification number: 0412.1 Document identification code: A

Keywords: gravitational wave; chemical element; synchrotron radiation; hadron; hydrogen; magnet

OCIS codes: 020.2070 Effects of collisions; 020.1335 Atom optics; 260.2110 Electromagnetic optics; 260.2160 Energy transfer

Fund projects, none

E-mail: wen7879@126.com;

\section{Introduction}

\section{Academic speculation related to gravitational waves}

\section{1 Earth also has magnetism}

The magnetism of magnets is the most important mechanics in every scientific field we know. Until now, humans have not yet known how the repulsive force and the gravitational force and the magnetic force and the universal 
gravitational force inside the atom are formed. Only their macroscopic physical properties are known, but there are inextricable connections between them. For example, magnetic force can change electricity, and electricity can change magnetic force. The formation of universal gravitation is even more unknown. Some scientists have proposed that as long as you understand quantum gravity, you can know how various forces in nature are formed. The Earth also has a magnetic force. This article speculates that this has an important relationship with universal gravitation, and this force is a changed force, which is related to the extreme conditions of high-temperature and high-temperature nuclear fusion inside the earth. Because all planets in the universe have nuclear fusion, scientists know that gravitation is related to mass and distance, and the physical properties of magnetic force are also very similar.

\subsection{Gravity between objects is not only related to mass}

Scientists believe that the gravitational force between two objects on the surface of the earth is only related to mass, and this article believes that there are other possibilities. The magnitude of neutron stars in the universe is large enough. If they explode, they will crack into many fragments, some of which are very small. But if their mass is astonishing, will it cause the surrounding planets and stars to move in a circular motion and re-form for a galaxy, there is no such phenomenon in cosmological cognition. Only the internal fusion and other extreme physical planets in the universe will drive the circular motion of other planets. Dark energy black holes and physical black holes are clear examples. Dark energy black holes finally form various galaxies, and the physical black hole is massive enough that it stops moving. Therefore, the formation of universal gravitation is not very important in relation to quality, but a force disguised in special conditions and the environment. Magnetic force is the most prominent and human-like force property of gravity. This paper intends to use magnetic force to do a quantum gravity experiment to verify the results. The dark energy formula is derived by simulation, and it is not absolutely correct. It is only for reference.

\subsection{High-energy particles have a lot to do with gravitation}

Cosmic media, photons, high-energy particles, rays, leptons, mesons. The study found that various rays in the universe, high-energy particles and gravitation are strongly connected. In 2017, for the third time, scientists detected gravitational wave signals from the merger of two cosmic black holes, which confirmed the existence of Einstein's general theory of relativity. (2) When the cosmic star was first formed, a cloud of hydrogen gas formed various elements after nuclear fusion. When the fusion fuel hydrogen gas was burned, gravitation collapsed into a black hole, and the formation of black hole caused space-time distortion. (1) Since universal gravity can be changed by physical means Strength, this article will try to analyze the physical connection between gravity and all aspects.

\section{2 basic principles}

\section{Analysis of two models of excited gravity}


Excitation and the use of the gravitational field, the method 1 synthetic heavy chemical element, make element quality his little black hole, gravitational field, space and time distortion, control increased, dark energy, because the gravitation closely connected with dark energy, modern physics experiment confirmed that excite gravity method 2 , like Einstein's general theory of relativity, space-time distortion within the space of the earth, the physical method to change the magnet or electric wire of atomic internal structure, as far as possible let magnet magnet magnetic field and the metal coil of the electric field force, expansion far field intensity range, to expand, expanding field line, if the field line string is the solar system, etc., Evolve into universal gravitation physical properties, chemical properties of the same force and field, the force of the same nature interact with each other, in the universe the magnitude of the gravitational force is greater than the earth's gravity, in the balance Angle of the force, using this principle to create spacecraft, can fly freely in space. Magnetism and electricity, the nuclear force is the force that we think of, and the force that exists in the universe, so we experiment with it. Unified field theory studies from electromagnetic field, nuclear force and gravitational field. Unified field theory holds that when any object in the universe is at rest relative to us, the space around it has a velocity $\mathrm{C}$ of light. Radiative motion, and therefore a body has a special resting momentum m'c, when the body is moving with a velocity $\mathrm{V}$ relative to us, its momentum is $\mathrm{m}(\mathrm{C}-\mathrm{v})$.

Nuclear force, electromagnetic force and universal gravitation surface are the interaction forces between objects. Essentially, they are all caused by the changes of matter points in space relative to the motion state of our observer. They are all inertial forces and the rate of change of momentum $\mathrm{P}=\mathrm{m}(\mathrm{c}-\mathrm{v})$ with time $\mathrm{t}$.

$$
\begin{gathered}
\mathrm{F}=\mathrm{dP} / \mathrm{dt}=\mathrm{Cdm} / \mathrm{dt}-\mathrm{Vdm} / \mathrm{dt}+\mathrm{mdC} / \mathrm{dt}-\mathrm{mdV} / \mathrm{dt} \\
(\mathrm{C}-\mathrm{V}) \mathrm{dm} / \mathrm{dt}=\mathrm{Cdm} / \mathrm{dt}-\mathrm{Vdm} / \mathrm{dt}
\end{gathered}
$$

According to unified field theory, it is the force of mass changing with time, and it is the electromagnetic force. $\mathrm{Cdm} / \mathrm{dt}$ is the electric field force, $\mathrm{Vdm} / \mathrm{dt}$ is the magnetic field force,

The inertial force in $\mathrm{mdV} / \mathrm{dt}$ in Newton's second theorem is also universal gravitation, and $\mathrm{mdC} / \mathrm{dt}$ is the nuclear force. Want to have a particle relative to the observer we rest, o space around an arbitrary point space geometry (in order to describe the movement of the space itself, we have divided our infinite space into many small pieces, each piece is called space geometric point, hereinafter referred to as geometric point) $\mathrm{p} \mathrm{C}$ at light speed in zero time, this paper argues that the speed of light to the vector, the speed of light as a vector direction can change) starting from point o, along a certain direction, experienced a time $\mathrm{t}$, in $\mathrm{t}$ 'moment will arrive at the location of the $\mathrm{p}$, let xyzo point $\mathrm{o}$ in rectangular coordinate system origin, point to point $\mathrm{p}$ radius vector by point $\mathrm{o} \mathrm{R}=\mathrm{C}$.

$$
t=x i+y j+z k
$$

$\mathrm{R}$ is a function of the spatial position $\mathrm{x}, \mathrm{y}$ and $\mathrm{z}$, which varies with the change of $\mathrm{x}, \mathrm{y}$ and $\mathrm{z}$, denoted as: 


$$
\mathrm{R}=\mathrm{R}(\mathrm{x}, \mathrm{y}, \mathrm{z},) .
$$

We enclose the particle $o$ as a Gaussian sphere $s=4 \pi r^{2}$ (the volume of the inner sphere is $4 \pi r^{3} / 3$ ) with the radius $\mathrm{R}=\mathrm{Ct}$ which $\mathrm{r}$ is the length $\mathrm{R}$ in the $\mathrm{Ct}$.

The gravitational field $\mathrm{A}$ around point $\mathrm{o}$ represents the displacement vector $\mathrm{R}=\mathrm{Ct}$ of $\mathrm{n}$ geometric points in the volume $4 \pi r^{3} / 3$ around point $o$,

$$
\mathrm{A}=\mathrm{g}(3 \mathrm{kn} / 4 \pi) \mathrm{R} / \mathrm{r}^{3}=\mathrm{g} \mathrm{mR} / \mathrm{r}^{3}
$$

$\mathrm{k}$ is the constant of proportionality. $\mathrm{g}$ is the gravitational constant.

The mass $\mathrm{m}$ of the mass point o represents the ratio of the number $\mathrm{n}$ of the geometric point vector displacement $\mathrm{R}=$ $\mathrm{Ct}$ and the solid angle $4 \pi$ within the Gaussian sphere $s=4 \pi r^{2}$ (the volume of the inscribed sphere is $4 \pi r^{3} / 3$ ).

$$
\mathrm{m}=3 \mathrm{kn} / 4 \pi
$$

Thus, the above gravitational field equation $\mathrm{A}=\mathrm{g}(3 \mathrm{kn} / 4 \pi) \mathrm{R} / \mathrm{r}^{3}$ can be written as:

$$
\mathrm{A}=\mathrm{g} \mathrm{mR} / \mathrm{r}^{3}
$$

Newton's law of universal gravitation states that the gravitational field $\mathrm{a}=\mathrm{gm} / \mathrm{r}^{2}$ generated at the space $\mathrm{p}$ around the particle $\mathrm{o}$ (the radius of the vector from o to point $\mathrm{p}$ is $\mathrm{R}$, the distance from point $\mathrm{o}$ to point $\mathrm{p}$, that is, the number of vector $\mathrm{R}$ is $\mathrm{r}$ ), Vector formula:

$$
\mathrm{A}=\mathrm{g} \mathrm{mR/ \textrm {r } ^ { 3 }}
$$

The above gravitational field equation and Newtonian mechanics gravitational field equation are consistent.

In the mass equation introduced above $\mathrm{m}=3 \mathrm{kn} / 4 \pi$, the angle is a constant $4 \pi$. In fact, the angle can be a variable, varying between 0 and $4 \pi$. Both $n$ and $m$ can be variables, and the mass equation still holds.

We introduce the concept of solid angle $\Omega$ and write the mass equation $m=3 \mathrm{kn} / 4 \pi$ in a general form:

$$
\mathrm{m}=\mathrm{kn} / \Omega
$$

Correspondingly, there are more general gravitational field equations:

$$
\mathrm{A}=\mathrm{gmR} / \mathrm{r}^{3}=\mathrm{gknR} / \Omega \mathrm{r}^{3}
$$

The corresponding Gaussian surface is

$$
\mathrm{s}=\Omega \mathrm{r}^{2}
$$

In the unified field theory, the electric field $\mathrm{E}$ generated by a particle $\mathrm{o}$ with a mass of $\mathrm{m}$ at the surrounding space $\mathrm{p}$ is defined as the change of the mass $m$ in the gravitational field with time $t$.

That is

$$
\mathrm{E}=\mathrm{gk} \mathrm{k}^{\prime}(\mathrm{dm} / \mathrm{dt}) \mathrm{R} / \mathrm{r}^{3}=\mathrm{qR} / \varepsilon \mathrm{c} 4 \pi \mathrm{r}^{3}
$$

$\mathrm{g}$ and $\mathrm{k}^{\prime}$ are constants, and $\varepsilon \mathrm{o}$ is the vacuum dielectric constant. 
Humans have discovered that when charged particles move at a speed V relative to our observer, they can cause a change in the electric field in the vertical direction of $\mathrm{V}$. The part of the electric field change can be regarded as a magnetic field, that is, the electric field that changes with the speed produces a magnetic field. Field theory inherits this view.

Imagine a point $\mathrm{o}$, which is stationary relative to our observer, with a mass of $\mathrm{m}$ and a charge of $\mathrm{q}$. An electrostatic field $\mathrm{E}$ is generated in the surrounding space $\mathrm{p}$. The radius of the vector from point o to point $\mathrm{p}$ is $\mathrm{R}$, which is generated by point $\mathrm{p}$ The electric field $\mathrm{E}$ is:

$$
\mathrm{E}=\mathrm{qR} / 4 \pi \varepsilon \text { or }^{3}=\mathrm{k}^{\prime}(\mathrm{dm} / \mathrm{dt}) \mathrm{R} / 4 \pi \varepsilon \text { or }^{3}
$$

$\mathrm{K}^{\prime}$ is a constant.

When point o moves relative to us at a speed $\mathrm{V}$, it can cause a change in the electric field $\mathrm{E}$. The part of the change can be regarded as the magnetic field $\mathrm{B}$. The simple idea is that the electric field $\mathrm{E}$ multiplied by the speed $\mathrm{V}$ is the magnetic field B. Because of the speed V and the electric field When E is perpendicular to each other, the generated magnetic field is the largest, so there is a cross product between them, so there is the following relationship,

$$
\mathrm{B}=\text { constant multiplied by }(\mathrm{V} \times \mathrm{E})
$$

From the geometrical equation of the electric field $\mathrm{E}$

$$
\mathrm{E}=\mathrm{q} \mathrm{R} / 4 \pi \varepsilon \text { or }^{3}=\mathrm{k}(\mathrm{dm} / \mathrm{dt}) \mathrm{R} / 4 \pi \varepsilon \text { or }^{3},
$$

The geometric form equation of magnetic field B can be obtained,

$$
\begin{aligned}
& B=\text { constant multiplied by }\left[\begin{array}{llllll}
V & \times(q R / 4 \pi & \varepsilon & \circ & r^{3}
\end{array}\right] \\
& =\text { constant multiplied by }\left[\mathrm{V} \times \mathrm{k}^{\prime}(\mathrm{dm} / \mathrm{dt}) \mathrm{R} / 4 \pi \quad \varepsilon \circ \mathrm{r}^{3}\right]
\end{aligned}
$$

Combining the constants, because we are discussing here in a vacuum, the above constant related to the magnetic field B uses the vacuum permeability $\mu$. representation

$$
B=\mu o\left[\begin{array}{lll}
V & \times k^{\prime}(d m / d t) R / 4 \pi & r^{3}
\end{array}\right]
$$

The above is the geometric form equation of the magnetic field in vacuum. This equation is closely related to the equation $\mathrm{B}=\mathrm{V} \times \mathrm{E} / \mathrm{c}^{2}$ that is satisfied by the relationship between the electric field and the magnetic field.

$$
\begin{aligned}
\mathrm{B} & =\mu \mathrm{o}\left[\mathrm{V} \times \mathrm{k}^{\prime}(\mathrm{dm} / \mathrm{dt}) \mathrm{R} / 4 \pi \mathrm{r}^{3}\right] \\
& =\mu \mathrm{o}\left[\mathrm{V} \times\left(\mathrm{qR} / 4 \pi \mathrm{r}^{3}\right)\right] \\
& =\mu \mathrm{o}\left[\mathrm{V} \times \varepsilon \mathrm{o}\left(\mathrm{qR} / 4 \pi \varepsilon \circ \mathrm{r}^{3}\right)\right] \\
& =\mu o \varepsilon \mathrm{O}\left[\mathrm{V} \times\left(\mathrm{qR} / 4 \pi \varepsilon \circ \mathrm{r}^{3}\right)\right] \\
& =\mu o \varepsilon \mathrm{o}(\mathrm{V} \times \mathrm{E})
\end{aligned}
$$

In electromagnetics, it is considered that the magnetic permeability in vacuum is $\mu \circ$. And the dielectric constant 
$\varepsilon \circ$. The product of is the reciprocal of the square of the speed of light $\mathrm{c}$ in vacuum (this is artificially specified), so the above equation can be written as:

$$
\mathrm{B}=\mathrm{V} \times \mathrm{E} / \mathrm{c}^{2}
$$

The above equation reflects the basic relationship between electric field and magnetic field. From this equation plus the space-time homogenization equation $\mathrm{r}^{2}=\mathrm{c}^{2} \mathrm{t}^{2}$, it can be derived that Maxwell' $\mathrm{s}$ equations are variable magnetic fields to produce electric fields and variable electric fields to produce magnetic fields.

Note that the above magnetic field and moving electric field do not consider the relativistic effect, but only hold when $\mathrm{V}$ is very small or equal to zero.

The electric field E generated when the charge moves should be multiplied by the relativistic correction phase $\Psi$ $=\left(1-\mathrm{v}^{2} / \mathrm{c}^{2}\right) /\left\{\sqrt{ }\left[1-\left(\mathrm{v}^{2} / \mathrm{c}^{2}\right) \sin ^{2} \theta\right]\right\}^{3}$, where $\theta$ is the geometric point displacement $\mathrm{R}$ and the $\mathrm{x}$-axis Angle. The electric field equation multiplied by the relativity correction phase $\Psi$ does not affect the relationship between the electric field and the magnetic field.

In unified field theory, mass and gravitational field are defined in this way. The universal gravitational field, electric field, and nuclear force field exist when the particles are at rest.

Gravity is proportional to acceleration and mass, and is the main force between objects with large mass and low velocity.

The force of the electromagnetic field is proportional to the change of mass over time, and the change of mass depends on the speed, and has nothing to do with the acceleration and the size of the mass. Therefore, the electromagnetic field force is the main force between objects with small mass and high speed.

The nuclear force is the force that changes the direction of the speed of light, and is related to the motion state of the particles being acted upon, so it is more complicated.

Below we use the basic electromagnetic field relationship $\mathrm{B}=\mathrm{V} \times \mathrm{E} / \mathrm{c}^{2}$ combined with the gravitational field combined with the definition equation to derive the mathematical relationship between the changing magnetic field and the gravitational field.

We still use the previous o point as the object of description. When o point is stationary relative to our observer, there is a geometric point $\mathrm{p}$ in the surrounding space, and o point generates an electrostatic field E' at point $\mathrm{p}$.

When point o moves at speed $\mathrm{V}_{\mathrm{x}}$ (for simplicity of description, we specify $\mathrm{V}$ along the $\mathrm{x}$ axis) relative to our observer, a magnetic field B is also generated at point o at $\mathrm{p}$, and:

$$
\mathrm{B}=\mathrm{V} \times \mathrm{E} / \mathrm{c}^{2}
$$

We assume that the electric field $\mathrm{E}$ does not change with time $\mathrm{t}$, and we obtain the partial derivative of the magnetic 
field $\mathrm{B}$ and velocity $\mathrm{V}$ with respect to time $\mathrm{t}$ in the above formula, and the result is:

$$
\partial \mathrm{B} / \partial \mathrm{t}=(\partial \mathrm{V} / \partial \mathrm{t}) \times \mathrm{E} / \mathrm{c}^{2}
$$

There are results:

$$
\begin{gathered}
\partial \mathrm{B}_{\mathrm{x}} / \partial \mathrm{t}=0 \\
\partial \mathrm{B}_{\mathrm{y}} / \partial \mathrm{t}=-\left(\partial \mathrm{V}_{\mathrm{x}} / \partial \mathrm{t}\right) \mathrm{E}_{\mathrm{z}} / \mathrm{c}^{2} \\
\partial \mathrm{B}_{\mathrm{z}} / \partial \mathrm{t}=\left(\partial \mathrm{V}_{\mathrm{x}} / \partial \mathrm{t}\right) \mathrm{E}_{\mathrm{y}} / \mathrm{c}^{2}
\end{gathered}
$$

According to the previous analysis, the gravitational field can be regarded as the acceleration of a geometric point $\partial \mathrm{V} / \partial \mathrm{t}$. From the above equation, it can be seen that the changing magnetic field, electric field, and gravitational field are perpendicular to each other.
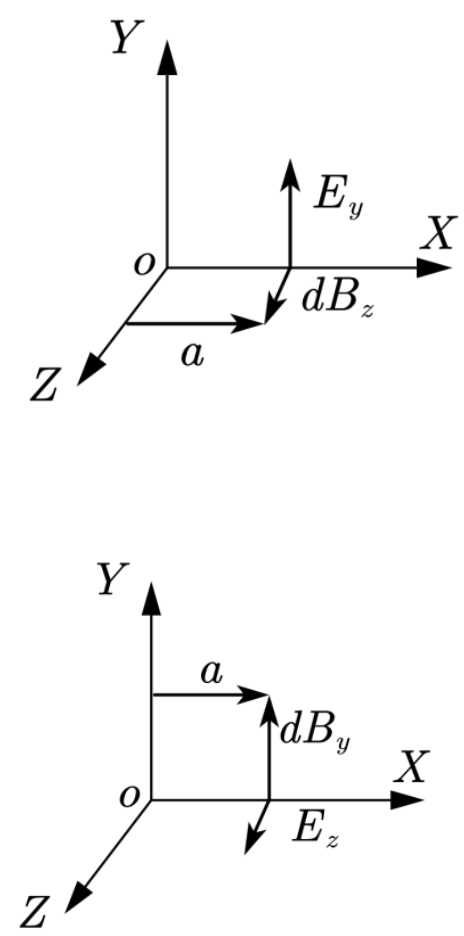

According to the description of the unified field theory, the Faraday electromagnetic induction principle is:

The changing magnetic field produces an electric field and a gravitational field, and the changing magnetic field, gravitational field, and electric field are perpendicular to each other.

However, the gravitational field generated by the changing magnetic field is a vortex field. Its curl is not zero, and its divergence is zero. Unlike the gravitational field generated by universal gravitation, which is distributed symmetrically around a point, the divergence is not zero, and the curl is zero. Therefore, the changing magnetic field cannot directly interact with the gravitational field. This is the reason why the world's use of changing electromagnetic fields to produce antigravity experiments failed. Gravitational field, nuclear force field and electromagnetic field have many things in common. If you want them to unify the experiment, extreme physics and extreme environment will make 
them qualitatively change. The same is true for the beginning and evolution of the universe.

\subsection{Simulation experiment of chemical elements precipitated by gravitational waves designed using the}

\section{principle of black holes}

If a simulation experiment is designed based on the principle of black hole formation, the realization of space-time distortion in earth space excites gravitational waves. The gravitational wave acting force is greater than the earth's gravity of $9.8 \mathrm{~N} / \mathrm{kg}$, and then escapes from the constraints of the earth and flies to other stars in the universe.

How to get excited gravitational waves in experiments? It needs to be performed under extreme physical conditions and special physical environments. The experiment of simulating the physical changes of stellar nuclear fusion may distort space-time (the hydrogen bomb is fusion, but the explosion time is too short to be space-time twisted). The experimental goal is to physically change the internal structure of the magnet and the atom of the metal coil to make the magnetic field of the magnet as much as possible. With the electric field of the metal coil, the gravitational field becomes larger. When the gravitational force reaches a certain level, it can interact with the forces of other planets in the universe. The gravitational wave strength and the proportion of the force are balanced to determine the spacecraft's flight direction. The nuclear fusion during the experiment radiates many rays: (including alpha rays, beta rays, gamma rays, infrared rays, ultraviolet rays, high-energy particles, etc.), and the products after nuclear fusion form a variety of chemical elements. (5) This confirms the experimental synthesis Elemental success rate is high.

The origin of the chemical elements of the universe has not been stated so far. This article intends to design experiments based on the extreme physical conditions of the universe. The formation of chemical elements in the universe is the product of stellar fusion (6), (3), (4). At the same time, various rays, such as infrared, gamma rays, x-rays, and ultraviolet rays, play an important role in chemical reactions. These rays are the physics of atomic nuclei. Changes radiate, indicating that they are strongly connected to the atomic structure. The experiment used high-density hydrogen, deuterium or tritium to represent the initial formation of hydrogen clouds. Alpha rays, $\mathrm{X}$ rays, infrared rays, ultraviolet rays, beta rays, gamma rays, neutron flow, proton flow, radiation and high-energy particles representing nuclear fusion radiation; an experiment is known that the radiation energy nuclear reaction equation, that is, a combination of neutrons and protons The deuteron emits 2.22 mega-electron volts of energy. This energy is radiated by gamma photons. In the opposite process, the tritium or deuterium nucleus is bombarded with gamma rays to be divided into neutrons and protons. Various types of radiation and counter-radiation provide energy for the synthetic elements. The energy includes dark energy (dark energy changes in the range where the value of space-time effect changes) and other different levels and different types of energy. In the experiment, there are neutron flow, proton flow, and $\beta$-ray (high-speed electron flow). Kind of ray, magnetic field (make the electron flow move farther around the circle), and quantum science knows that the 
main particles of atoms are neutrons, protons and electrons. As long as appropriate physical conditions are designed, artificial elements are not impossible. After the operation of our nuclear reactor, a large amount of waste is often generated. These wastes are not the original uranium, but various other radioactive materials. It can be seen that particles and rays collide with each other to form some chemical elements. The principle of simulation experiments, typical magnetically constrained nuclear fusion, but this process does not meet the actual nuclear fusion requirements. It is a subtype magnetically constrained nuclear fusion device, but it can also form a weak confinement space, free negative electrons (B Rays) and positive protons stay in a limited space. Because deuterium and tritium do not have nuclear fusion, but after passing in Y-rays, the deuterium and tritium react cyclically. This reaction is different from the quantum energy emitted by chemical reactions. This energy property is flexible quantum energy, which is closest to the nucleus Core energy level. Magnetically constrained nuclear fusion constrained space. X-rays, B-rays, neutron flow, proton flow and other particles collide with each other. The energy and pressure collapse and the compression density become larger. In the process, the rays provide the required conditions for the quark, and the energy is asymmetric., So as to achieve the quark effect and the weak electric phase transition effect in the universe.

\section{Analysis and discussion}

Hoyle scientists and others did not succeed in conducting nuclear fusion element experiments because they were too focused on nuclear binding energy to achieve the abundance of cosmic elements. In the process, they also lacked the supply of gluons required for the second-change reaction, which was unsuccessful and reached the element. The combined abundance is not necessarily thermal energy and negative temperature. There are various kinds of quantum energy. We cannot prevent the choice of special quantum ones to allow him to reach the cosmic abundance, such as r-ray energy and the deuterium-deuterium reaction energy mentioned above. The magnetic confinement nuclear fusion device is used to simulate the nuclear fusion environment and the gluons required for nuclear synthesis, so that its energy is infinitely asymmetric, so as to induce the gluons to combine into nucleons, thereby generating atoms. Why do I say that the space-time effect controls dark energy? Have you noticed that the heavier the weight of the nucleus, whether it is an atom, or a planet, or a black hole, the greater the gravitational force and the gravitational force increase hundreds of times? We use this feature to excite Gravity, the more the gravitational force surpasses the earth's first, second, and third speeds, it is possible to get rid of the constraints of the earth. Of course, some people think that I use both the binding energy and the abundance of the cosmic elements to make special quantum energy in the subtype magnetic confinement nuclear transformation device. Infinite asymmetry, the nucleus is separated into super-heavy proton nucleus or atom. These super-density nuclei have thousands, tens of thousands, hundreds of millions of protons, neutrons, and electrons combined into individual atoms, which are small black holes, infinitely variable in space and time. The larger, the more 
dark energy is controlled, the greater the gravity.

This experiment studies from four aspects: cosmic abundance; active ground state energy saturation; and infinite asymmetry of energy required by various elements; sufficient gluons required in quarks, and pressure. Every necessary condition in the nucleosynthesis element is satisfied. The failure of modern man-made chemical element experiments is because the four major factors must be indispensable.Energy pressure makes these hadrons, quanta, energy collide, collapse, turbulence, chaos, space makes all kinds of quanta need and supplement, and at the same time, it can timely supplement the basic particles and energy that disappeared in the lack of symmetry, so that the infinitely unsatisfied interface in the independent turbulence space can be saturated to a certain extent, which may cause space-time bending and gravitational field changes. This is the principle of designing this experiment.

4 Simulated experimental equipment method for obtaining artificial chemical elements using gravitational waves

This article intends to design an experimental device that excites gravitational waves and also an artificial chemical element device to verify the previous conjecture.

First, use a spherical object with a diameter of about 1 to 2 meters. The outer shell is made of metal and the inner layer is made of heat-insulating polymer material to prevent heat loss. A round ring magnet in the center of the circle has strong magnetism and weighs $4 \mathrm{~kg}$. On the left and right, a lead rod is tightly set in the center of the magnet. As a material for reflecting rays, the lead rod is applied with a voltage of more than 0.5 volts. Five round coils are added to the outside of the magnet. The diameter of the coil is $8 \mathrm{~cm}$, which can be larger., The coil applies a voltage between 0.5 volts and 220 volts. The coil uses a mixture of dozens of metal elements, and if it can use a part of actinide and lanthanide elements, and it can be used for fission with neutrons, it is not necessary to prevent nuclear fission. Note that the coil can only be made of metal, and the reaction between metal elements is not easy. Install unusually strong ultraviolet, infrared, alpha rays, beta rays, gamma rays, and x-ray emitters on the equator of the sphere; install two sets of emitters for each type of ray, and install two sets of each on the top of the sphere The principle of sub-stream emitter, proton-stream emitter, neutron-stream emitter, is emitted by the plutonium element. The products produced by alpha particles bombarding the plutonium plate are neutron flow, and bombarding paraffin with neutron flow is proton flow emitter, alpha ray, beta ray, gamma ray emitter, lead box releases thorium element, lead box strengthens magnetic field electric field, electrode Above the lead box, the rays emitted from the box are its. The inside of the sphere must be vacuum. The interior is filled with high-density hydrogen, a mixture of tritium and deuterium. Hydrogen is very important in this experiment because the star formation is a hydrogen cloud, and the universe the most widely existing gas, neutron flow, and $\gamma$-ray bombardment of hydrogen, this energy is the most important energy of synthetic elements, 
and is different from the energy of heat temperature. The whole process allows rays to collide with rays, rays collide with magnets and coils, neutrons and protons collide, and neutron and proton current experiment generators are carried out in a sphere, which generates a large number of leptons, media, etc. More favorable, leptons, mesons, etc. are all important for the formation of nuclei.

Based on the energy of the forming element, as long as the intensity of the rays such as gamma rays, $\mathrm{X}$ rays, and infrared rays is changed in this experiment, the time required to form the elements within a certain proportion of the intensity of the rays is adjusted, and the heat generated by the coil current and the magnetic force of the magnet are appropriately adjusted. Intensity, or the formation of gravitational waves with different elements and different intensities, can be verified specifically after real experiments. In the experiment, be very careful to protect the loss of plutonium element, otherwise radioactive elements will leak; pay attention to changes in the sphere, excessive pressure, high temperature, or other physical changes.

\section{Conclusions}

Benefits of this experiment: Rays, neutron currents, and proton currents bombard magnets, the coils undergo micro-hour space distortion, the excitation magnetic field and electric field evolve into force fields with the same physical properties of universal gravitation, and use it to make spacecraft, or to change the space combustion dynamics, and Instead of the current rocket power, flying in cosmic space can greatly reduce the cost, time and distance of aerospace, and play a huge role in studying various secrets of the universe. In the experiment, similar to the environment and conditions of the formation of various elements of the cosmic star, the formation of artificial elements is absolutely possible. And there are many man-made elements behind position 93 of the periodic table of chemical elements. These elements are extremely unstable in physical properties and can easily become other elements. Most of these elements are obtained by particle accelerators bombarding other substances, that is, target-type physics or quark physics, which form elements in a short time. This article speculates that the target-type and quark physical man-made elements have insufficient energy or lack of the class of unimportant but indispensable basic constituent particles may also be less important constituent particles, such as neutrinos, leptons, mesons, etc., whose element physical properties are unstable. Therefore, by designing the simulated nuclear fusion time under such extreme physical and special environmental conditions, after a long enough and intense collision simulation, or the synthesis of elements with stable physical properties and the discovery of new chemical elements, the technical complexity of the process of refining artificial elements, Difficult question.

After the discovery of gravitational waves, the curvature of space-time and dark energy were determined, and 
the gravitational effect of dark matter was discovered. The curvature of space-time may be the control of dark energy and dark matter. The only way for mass is that time and space bending is related to extreme physical strength and mass. The method of combining the source of cosmic elements with modern physics methods was deeply studied, and the experimental method of space elements was designed. If the theory and experiment being proved successful, it will open up new channels and models for the development of aerospace engineering and space \& chemistry research. This model will break the development of modern science and promote the development of astronomy, chemistry and physics. Great changes, the significance of science is inestimable.

Due to my limited cultural level and the difficulty of the experiment, the experiment is still in the stage of reasoning and imagination. I invite relevant experts to criticize and correct it.

\section{References}

[1] Ding Liang, Li Fangyu, Time-Space Perturbation Effects of High-Frequency Gravitational Waves in the Electromagnetic Ring Cavity and ToKamaK Focus Region, Chongqing University, 2010,

[2] Liu Jian, the first gravitational wave detection event GW150914 and gravitational wave astronomy, Science Bulletin, 2016-14

[3] Zhang Ning, Einstein's theory of relativity on the characteristics of time and space, Beijing Union University, 200501

[4] Ouyang Ziyuan, Celestial Chemistry, Advances in Earth Science, 1994.02

[5] Gu Linfu, the origin of chemical elements, Natural Science Journal of Heilongjiang University, 199203

[6] Xu Lanping, the origin of the problem of chemical elements, astronomy, 1987.01

[7] Deng Zuyi. Cosmology__ A Product of Human Civilization [J]. 1990 (2): 19-25.

[8] Yin Lan, Rao Yihua. Research on Magnetic and Hydrodynamics of Key Energy Conversion Components of Magnetically Binding Nuclear Fusion [J]. Science and Technology Information, 2014, 012 (022): 1.

[9] Lu Guanghong, Luo Guangnan, Li Jiangang. Research Progress on Plasma-Wall Interactions in a Magnetic Confinement Tokamak\%

[10] Research progress on interaction between Tokamak plasma and wall in constrained fusion [J]. Progress in Chinese Materials, 2010, 029 (007): 42-48.

[11] Cui Jianhua, Zhai Zhongxu, Zhang Tongjie. Dark energy abundance in the $\Lambda$ CDM universe model [J]. Journal of Dezhou University, 2011 (04): 7-10.

[23]Shi-Yong Chen,Ben-Wei Zhang,Enke Wang. Jet charge in high-energy nuclear collisions[J]. IOP Publishing,2020,44(2).

[13] Zhang Jiang, Cui Wenyuan, Zhang Bo. Research progress on the nuclear synthesis of metal-poor star $r$ process [J]. Advances in Astronomy, 2011 (04): 33-53.

[14] Liu Honglin, Luo Zhiquan. Interstellar (26) Al Nuclear Synthesis Research Progress [J]. Review of Nuclear Physics, 2008 (01): 33-39.

[15] Tang Xiaodong, Li Kuoang. The Origin of Elements in the Universe [J] .Physics, 2019,48 (10): 633-639.

[16] Shuhua, Zhou. Loop quantum gravity tracking black hole evolution [J] .Physics, 2019,48 (02): 91. 
[17] Chen S Y, Zhang B W, Wang E K. Jet charge in high energy nuclear collisions [J]. 2019.

[18] Wan Niu. Study on the Properties of Symmetric Energy Using Nuclear Decay [D]. 2019.

[19] Tang Xiaodong, Li Kuoang. The Origin of Elements in the Universe [J]. Physics, 2019, 48 (10): 633-639.

[20] Zhou Shuhua. Loop quantum gravity tracks black hole evolution [J]. Physics, 2019, 48 (02): 28.

[21]Di Wu,Chun-Lin Bai,H.Sagawa,Zeng-Qiang Song,Huan-Qiao Zhang.Contributions of optimized tensor interactions on the binding energies of nuclei[J].Nuclear Science and Techniques,2020,31(02):13-21.

[22] Wan Niu, Xu Chang. Using the decay of unstable nuclei to study the properties of symmetric energy of nuclear matter [J]. Chinese Science Bulletin, 2020, 65 (07):594-609. 